\title{
Narrativa
}

\section{Appunti sul rapporto tra questione meridionale e nazionale-popolare in Gramsci con una nota sul cinema di Matteo Garrone}

Paolo Desogus

\section{OpenEdition}

\section{Journals}

Edizione digitale

URL: https://journals.openedition.org/narrativa/654

DOI: $10.4000 /$ narrativa.654

ISSN: 2804-1224

Editore

Presses universitaires de Paris Nanterre

\section{Edizione cartacea}

Data di pubblicazione: 1 décembre 2017

Paginazione: $41-51$

ISBN: 978-2-84016-289-6

ISSN: $1166-3243$

Notizia bibliografica digitale

Paolo Desogus, «Appunti sul rapporto tra questione meridionale e nazionale-popolare in Gramsci con una nota sul cinema di Matteo Garrone», Narrativa [Online], 39 | 2017, online dal 01 décembre 2021 , consultato il 14 janvier 2022. URL: http://journals.openedition.org/narrativa/654 ; DOI: https://doi.org/ 10.4000/narrativa.654

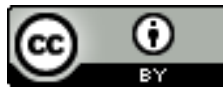

Narrativa est mise à disposition selon les termes de la Licence Creative Commons Attribution 4.0 International. 


\section{Appunti sul rapporto tra questione meridionale e nazionale-popolare in Gramsci con una nota sul cinema di Matteo Garrone}

\footnotetext{
$\longrightarrow$ uesto scritto nasce dal confronto tra la riflessione politica di Gramsci sul meridione d'Italia condotta nell'ultima parte della sua militanza politica come segretario del Pcd'I e gli studi successivi sul nazionale-popolare contenuti nei Quaderni del Carcere. Esso persegue due obiettivi: il primo è quello di mostrare il legame tra i modi e le forme attraverso i quali i temi della "disgregazione sociale del Mezzogiorno" e dell"“alleanza tra contadini e movimento operaio" - trattati, come è noto, nello scritto Alcuni temi della quistione meridionale ${ }^{1}$ - costituiscono una base decisiva della riflessione sul rapporto tra popolo, politica e cultura sviluppata successivamente da Gramsci in carcere. A partire dalla ricostruzione di questo legame e attraverso un breve cenno all'opera cinematografica di Matteo Garrone, il secondo obiettivo è invece quello di mostrare come la crisi della nozione di nazionale-popolare - dunque la crisi di quel pensiero che ha tentato di rendere sinonimi "nazione" e "popolo"2 - abbia contribuito al declino del meridionalismo italiano. In questa doppia prospettiva, questione del nazionale-popolare e questione meridionale verranno intese come reciprocamente coestensive, ovvero come termini che, pur indicando una serie di temi

1. Gramsci, Antonio, Alcuni temi della quistione meridionale, (1926), in ID., La costruqione del partito comunista. 1923-1926, Torino, Einaudi, 1971.

2. ' 'È da osservare il fatto che in molte lingue, 'nazionale' e 'popolare' sono sinonimi o quasi (così in russo, così in tedesco in cui 'volkisch' ha un significato ancora più intimo, di razza, così nelle lingue slave in genere; in francese 'nazionale' ha un significato in cui il termine 'popolare' è già più elaborato politicamente, perché legato al concetto di 'sovranità', sovranità nazionale e sovranità popolare hanno uguale valore o l'hanno avuto). In Italia il termine 'nazionale' ha un significato molto ristretto ideologicamente e in ogni caso non coincide con 'popolare', perché in Italia gli intellettuali sono lontani dal popolo, cioè dalla 'nazione' e sono invece legati a una tradizione di casta, che non è mai stata rotta da un forte movimento politico popolare o nazionale dal basso" (ID., Quaderni del carcere, Torino, Einaudi, 1975, q 21, \5, p. 2116).
} 
politici e culturali diversi, condividono alcuni tratti comuni, tra i quali il nesso tra cultura e popolo e soprattutto il rapporto tra subalternità e coscienza marxista.

Ora, con Alcuni temi della quistione meridionale, saggio lasciato incompiuto per via dell'arresto nel 1926, Gramsci si propone di "far uscire la quistione meridionale dalla fase indistinta [e] intellettualistica [...], per farla entrare in una fase nuova"3 ovvero nella fase della lotta politica organizzata. Rispetto alle indagini che pure hanno avuto un certo impatto sul pensiero gramsciano, come quelle di Gaetano Salvemini ${ }^{4}$ e di Benedetto Croce, si registra dunque un cambio di paradigma: occuparsi della questione meridionale non significa più fare del Mezzogiorno d'Italia un mero oggetto di analisi, ma lavorare affinché le sue forze divengano un soggetto politico, ovvero un agente di trasformazione sociale, culturale ed economica in grado di unirsi al movimento operaio internazionale. In questa prospettiva la preoccupazione principale di Gramsci è quella di dare soluzione alla frammentarietà delle lotte dei contadini del Mezzogionno e di tradurre in termini rivoluzionari le loro istanze, dando "espressione centralizzata alle loro aspirazioni e ai loro bisogni" . A questo scopo, dopo aver riflettuto sulla composizione sociale del lavoro in Italia, Gramsci indica come punto di partenza l'alleanza tra operai del Nord e contadini del Sud ${ }^{6}$. Il divario tra queste due aree del paese, come emerge anche nelle tesi congressuali di Lione, scritte insieme a Palmiro Togliatti nel gennaio dello stesso anno, ha il suo cuore nel Nord e più precisamente nel padronato industrializzato che, con la complicità dei grandi latifondisti del Sud, "dà alle popolazioni lavoratrici del Mezzogiorno una posizione analoga a quella delle popolazioni coloniali" " Una loro alleanza appare dunque indispensabile. In questa prospettiva, però, la classe operaia "deve spogliarsi di ogni residuo corporativo, di ogni pregiudizio o incrostazione

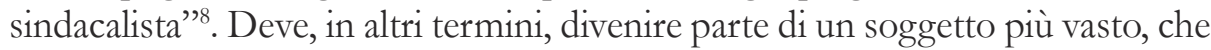
integra gli strati sociali subalterni:

Il metallurgico, il falegname, l'edile, ecc. devono non solo pensare come proletari e non più come metallurgico, falegname, edile, ecc., ma devono fare

3. ID., Alcuni temi della quistione meridionale, cit., p. 139.

4. Ibid., pp. 141-142.

5. Ibid., p. 150.

6. " $\Pi] 1$ concetto fondamentale dei comunisti torinesi non è stato la 'formula magica' della divisione del latifondo, ma quello della alleanza politica tra operai del Nord e contadini del Sud per rovesciare la borghesia dal potere di Stato" (Ibid., p. 139).

7. Gramsci, Antonio, Togliatti, Palmiro, La situazione italiana e $i$ compiti del Pci, (1926), in Gramsci, Antonio, La costruqione del partito comunista. 1923-1926, cit., p. 492.

8. Gramsci, Antonio, Alcuni temi della quistione meridionale, cit., p. 144. 
ancora un passo avanti. [...] Se non si ottiene ciò, il proletariato non diventa classe dirigente e questi strati che in Italia rappresentano la maggioranza della popolazione, rimanendo sotto la direzione borghese, dànno allo Stato la possibilità di resistere all'impeto proletario e di fiaccarlo?

In queste parole si riconoscono perfettamente i prodromi della nozione del nazionale-popolare che più tardi verrà sviluppata nei Quaderni del carcere e che occupa una posizione cruciale nel pensiero politico di Gramsci. Anche se in forma non pienamente compiuta, la convergenza tra operai e contadini viene infatti descritta non come una semplice convergenza strategica, ma come un'unione organica entro un quadro politico al contempo nazionale e internazionale. Sia nelle Tesi di Lione che in Alcuni temi della quistione meridionale è infatti molto forte l'idea che i lavoratori debbano superare la propria dimensione particolare, debbano appunto fare un "passo avanti" e inaugurare un percorso di lotte nazionali capace di portare anche in Italia le trasformazioni della Rivoluzione d'Ottobre.

Si avrà modo di valutare più avanti la pregnanza politica e filosofica della metafora del "passo avanti". $\grave{E}$ in prima istanza opportuna una digressione sul tema del sindacalismo menzionato da Gramsci. Per quanto nel saggio del '26 sulla questione meridionale appaia confinato a un breve paragrafo, esso costituisce un argomento di riflessione di cui si ha una prima traccia nel 1918 e che ciclicamente ritorna in molti altri scritti ${ }^{10}$ per poi essere ripreso nei Quaderni. Nel 1926, scrive Gramsci, "Il sindacalismo", è inteso come "l'espressione istintiva, elementare, primitiva, ma sana, della reazione operaia contro il blocco [della] borghesia""11. Esso costituisce una forma di lotta spontanea, dal basso, che tuttavia non è capace di delineare uno scenario di trasformazione complessiva dei rapporti tra capitale e lavoro. Il suo limite, come spiega nei Quaderni, è quello di ridurre il conflitto a un "rapporto fondamentalmente economico sociale"12, come la lotta per il salario, il posto di lavoro ${ }^{13}$ o anche per l'appropriazione della terra: tutti obiettivi nobili, necessari, ma che sul piano più strettamente politico

9. Ibid., p. 145.

10. Cfr. ID., "L'organizzazione economica ed il socialismo", in Il Grido del popolo, n. 707, 9 febbraio 1918, in ID., La città futura. 1917-1918, Torino, Einaudi, 1982, pp. 644-646; "La conquista dello stato", in L'Ordine Nuovo, a. I, n. 9, 12 luglio 1919, in ID., L’Ordine Nuovo, 1919-1920, Torino, Einaudi, 1987, pp. 127-133; "Discorso agli anarchici”, a. I, n. 43, 3-10 aprile 1920, in L'Ordine Nuovo, 1919-1920, cit., pp. 487-492.

11. Gramsci, Antonio, Alcuni temi della quistione meridionale, cit., p. 146.

12. ID., Quaderni del carcere, cit., q. 13, \17, p. 1581.

13. Ibid., q. 15, \39, pp. 1976-1977. 
non consentono ai gruppi sociali di sconfiggere gli oppressori e di divenire classe egemone. Anche nella sua forma più avanzata e rivoluzionaria, il sindacalismo condivide infatticonil liberalismola medesima matrice dell'"economismo", il quale, secondo Gramsci, considera i processi economici come la mera espressione della società civile ${ }^{14}$.

Si tratta di un punto evidentemente cruciale che, come ha mostrato recentemente Michele Prospero, tocca uno degli aspetti più importanti, nonché più attuali, della scienza politica di Gramsci, ovvero quello del rapporto tra economia e politica ${ }^{15}$. Per il pensatore sardo non esiste indirizzo economico che non sia frutto di una scelta politica. Non esiste, anzi, un'autonomia dell'economia. Anche il liberismo puro, che come è noto professa la totale separazione tra stato ed economia, è un indirizzo politico ${ }^{16}$. Si tratta di una valutazione che trova del resto riscontro anche in alcuni settori del pensiero neomarxista. Per esempio, Dardot e Laval, due fra gli autori oggi più discussi, pongono al centro dei loro studi l'importanza che gioca lo stato nella concezione economico-politica del neoliberismo attuale ${ }^{17}$. Qualsiasi soggetto antagonista - ieri come oggi - non può dunque muoversi esclusivamente nella sfera della società civile come proponeva il sindacalismo e come oggi tende a fare il movimentismo che rifiuta l'organizzazione politica; il "passo avanti" che deve svolgere è quello di superare la concezione liberale che tiene questo ambito separato da quello della sfera politica, allo scopo di candidarsi a diventare forza egemone in grado di esprimere una propria concezione dello stato.

Come è noto, nei Quaderni, per dare una prospettiva storica alle lotte contadine e operaie, Gramsci pone al centro della sua riflessione il partito politico, da lui inteso come corpo intermedio che per la sua natura anfibia tiene insieme società politica e società civile. Rispetto alle Tesi di Lione, la novità di questa riflessione è quella di superare l'idea di partito leninista come avanguardia capace di guidare il proletariato verso la rivoluzione e la trasformazione radicale della società. In Gramsci il partito politico è concepito non come una guida, ma come un intellettuale collettivo, come l'agente che propizia quell'unione

14. Ibid., q. 13, \18, pp.1589-1597.

15. Cfr. Prospero, Michele, La scienza politica di Gramsci, Roma, Bordeaux, 2016, pp. 110-122.

16. Oltre alla già citata nota $\int 18$ del quaderno 13 , cfr. inoltre q. $6, \int 10$, p. 692 , e q. 10 , \20, p. 1258.

17. Dardot, Pierre, Laval, Christian, La Nouvelle raison du monde. Essai sur la société néoliberale, Paris, La Découverte, 2010. 
organica tra gruppi dirigenti e popolo chiamata "blocco storico" in cui struttura e sovrastruttura trovano unità organica.

Se il rapporto tra intellettuali e popolo-nazione, tra dirigenti e diretti, tra governanti e governati, è dato da una adesione organica in cui il sentimentopassione diventa comprensione e quindi sapere (non meccanicamente, ma in modo vivente), solo allora il rapporto è di rappresentanza, e avviene lo scambio di elementi individuali tra governati e governanti, tra diretti e dirigenti, cioè si realizza la vita d’insieme che sola è la forza sociale, si crea il "blocco storico"18.

In questa prospettiva, la riflessione sulla forma partito va di pari passo col lavoro politico di costruzione del referente sociale antagonista che, soprattutto nel Mezzogiorno, pone problemi e questioni esemplari per la difficoltà di realizzare il passaggio dal sentire al comprendere e dal comprendere al sapere. Come già emerge con chiarezza in Alcuni temi della quistione meridionale, nelle popolazioni del Sud Gramsci individua un senso comune incapace di divenire coscienza progressiva, filosofia del riscatto. Sottoposta all'egemonia dei grandi possidenti, la concezione del mondo espressa dai contadini del Mezzogiono appare lacerata, disorganica, prepolitica, animata dal fatalismo e soprattutto marginalizzata dagli intellettuali che vivono in una dimensione cosmopolita, distaccata cioè dai problemi locali e nazionali. In termini marxiani queste popolazioni contadine sono classe in sé, ma non per sé ${ }^{19}$. Sono, in altri termini, una classe da un punto di vista oggettivo, data la condizione sociale in cui versano. Non sono invece una classe sociale da un punto di vista soggettivo perché esse non esprimono né una coscienza politica antagonista, né possiedono degli intellettuali in grado di suscitarla ${ }^{20}$.

Nel pensiero dei Quaderni quella del meridione diviene l'esperienza politica e insieme culturale attraverso la quale riflettere sui modi e le forme validi per restituire una soggettività ai subalterni. Tanto più essi restano lontano dalla formazione di una coscienza storico-politica, tanto più la loro esistenza sarà

18. Ibid., q. 11, \67, p. 1505.

19. Cfr. Marx, Karl, Misère de la philosophie, Paris-Bruxelles 1847, tr. it., Miseria della filosofia, in MArx, Karl, ENGELs, Friedrich, Opere complete, vol. VI, Roma, Editori Riuniti, 1973, p. 224.

20. "Si può dire che l'elemento della spontaneità è perciò caratteristico della 'storia delle classi subalterne' e anzi degli elementi più marginali e periferici di queste classi, che non hanno raggiunto la coscienza della classe 'per sé' e che perciò non sospettano neanche che la loro storia possa avere una qualsiasi importanza e che abbia un qualsiasi valore lasciarne tracce documentarie" (GrAmsci, Antonio, Quaderni del carcere, cit., q. 3, $\int 48$, p. 328). 
determinata meccanicisticamente dai rapporti di produzione ${ }^{21}$. Alla luce della riflessione sul blocco storico e sul partito politico, il loro riscatto non consiste più solo nel superamento di una condizione di sopraffazione, ma esso prefigura anche il momento di costruzione di un'egemonia capace di esprimere una nuova civiltà, un nuovo modo di vivere e sentire il mondo. Per Gramsci, infatti, la classe diviene protagonista dei processi storici - diviene classe per sé, e fa "un passo avanti" - modificando i rapporti di produzione e parallelamente - in un senso che ricorda per molti versi Benjamin - facendosi portatrice di una propria tradizione, di una propria cultura $a^{22}$.

In questa prospettiva storico-politica, la cultura e le arti sono chiamate a interpretare un ruolo cruciale, se non addirittura fondativo, poiché finalizzato non soltanto a unire gruppi sociali subalterni, ma a mediare il sentire dei subalterni e a trasformarlo in comprensione e dunque in sapere che li libera dalla passività e dal fatalismo meccanicistico. Non deve per questo stupire se il tema del nazionale-popolare, benché la sua portata sia molto più ampia, venga trattato maggiormente proprio nelle note sulla letteratura. Insieme alle arti, la letteratura partecipa al processo di costruzione della coscienza di classe. L'espressione artistica non si limita infatti a rappresentare la realtà: essa dà forma simbolica all'umano, ai suoi sentimenti, alle sue concezioni e alle sue aspirazioni. In questo modo essa è il cemento che consente di saldare il popolo alla nazione, le aspirazioni dei subalterni alla prospettiva di trasformazione dei rapporti di produzione.

Si badi però che per Gramsci lo scrittore e l'artista non debbono essere necessariamente organici al partito dei subalterni. Nelle sue note, egli prende in esame esperienze letterarie estranee al materialismo storico seguendo in questo modo lo stesso procedimento adottato da Marx ed Engels nella lettura di Balzac. Un esempio di scrittore nazionale-popolare è per Gramsci Dostoevskij, dunque uno scrittore conservatore, che ha abbracciato il socialismo solo per un breve periodo della gioventù ${ }^{23}$. Nei Quaderni non viene infatti mai adombrata

21. "Si può osservare come l'elemento deterministico, fatalistico, meccanicistico sia stato un 'aroma' ideologico immediato della filosofia della prassi, una forma di religione e di eccitante (ma al modo degli stupefacenti), resa necessaria e giustificata storicamente dal carattere 'subalterno' di determinati strati sociali’ (Ibid., q. 11, $\$ 12$, pp. 1387-1388).

22. Benjamin, Walter, Über den Begriff der Geschichte, (1940), tr. it., Sul concetto di storia, in ID., Opere complete. VII. Scritti 1938-1940, Torino, Einaudi, 2006.

23. A proposito del grande scrittore russo Gramsci scrive: "In Dostojevschij c’è potente il sentimento nazionale-popolare, cioè la coscienza di una missione degli intellettuali verso il popolo, che magari è 'oggettivamente' costituito di 'umili' ma deve 
la necessità di una letteratura di partito, né vengono definiti i criteri per un'estetica marxista. Il pensiero gramsciano, in quanto filosofia della prassi - filosofia che cerca un punto di mediazione tra pensiero e lotta politica - si occupa certamente di letteratura, le conferisce un ruolo centrale per la conquista dell'egemonia e per la costruzione del blocco storico, dà inoltre molto spazio alla critica e al ruolo dell'intellettuale come organizzatore di cultura e come punto di incontro tra arte e popolo, ma non stabilisce ricette.

Proprio per queste sue caratteristiche, la pubblicazione delle opere di Gramsci ha avuto nel secondo dopoguerra un impatto straordinariamente fecondo, anche perché capaci di dare risposte e di indirizzare i soggetti politici dell'epoca. I suoi Quaderni vengono dati alle stampe negli anni che seguono la lotta di Liberazione, la tentata riforma agraria di Fausto Gullo, la Costituente e la nascita dei partiti di massa, tutti eventi direttamente legati a quella che Ernesto de Martino, con una formula molto efficace, ha chiamato l'“irruzione nella storia del mondo popolare subalterno" 24 .

A raccogliere la testimonianza di questa irruzione, oltre allo stesso de Martino, vi sono stati numerosi scrittori, registi, artisti, antropologi, storici che in modi diversi si sono richiamati a Gramsci o che la critica ha studiato attraverso il pensiero dei Quaderni ${ }^{25}$. Il problema della letteratura e delle arti assume in questa fase un carattere politico soprattutto nelle fila del partito di cui Gramsci è stato tra i fondatori nonché primo segretario, cioè il Partito comunista italiano $^{26}$. L'eredità di quell'esperienza storico-culturale è certo problematica e controversa, specie nella lacerante polemica tra Elio Vittorini e Togliatti,

essere liberato da questa 'umiltà', trasformato, rigenerato. Nell'intellettuale italiano l'espressione di 'umili' indica un rapporto di protezione paterna e padreternale, il sentimento 'sufficiente' di una propria indiscussa superiorità, il rapporto come tra due razze, una ritenuta superiore e l'altra inferiore, il rapporto come tra adulto e bambino nella vecchia pedagogia o peggio ancora un rapporto da 'società protettrice degli animali', o da esercito della salute anglosassone verso i cannibali della Papuasia" (GRAMSCI, Antonio, Quaderni del carcere, cit., q. 21, \$ 3, p. 2112).

24. De MARTino, Ernesto, "Intorno a una storia del mondo popolare subalterno", in Società, a. V, n. 3, settembre 1949, ora in Clemente, Pietro, Meoni, Maria Luisa, SQuillacСІотті, Massimo, Il dibattito sul folkelore in Italia, Milano, Edizioni di cultura popolare, 1976.

25. Sulla ricezione di Gramsci rimandiamo a Liguori, Guido, Gramsci conteso. Interpretazioni, dibattiti e polemiche. 1922-2012, Roma, Editori Riuniti, 2012. Per quanto riguarda l'ambito più strettamente critico-letterario, cfr. GATTO, Marco, Nonostante Gramsci. Marxismo e critica letteraria nell'Italia del Novecento, Macerata, Quodlibet, 2016.

26. VITTORIA, Albertina, Togliatti e gli intellettuali. La politica culturale dei comunisti italiani (1944-1964), Roma, Carocci, 2014. Nonostante i progressi della ricerca storica, resta inoltre utile il confronto con Ajello, Nello, Intellettuali e Pci. 1944-1958, Bari, Laterza, 1979. 
o nell'inconcludente disputa tra Carlo Salinari e Carlo Muscetta su Metello di Vasco Pratolini ${ }^{27}$, o ancora nella clamorosa incomprensione proprio ai danni di de Martino ${ }^{28}$. Resta il fatto che mai come in nessun altro momento della storia italiana le classi popolari, e in particolare le classi popolari del Mezzogiorno, hanno goduto in Italia di una così larga attenzione.

Quella saldatura tra intellettuali e popolo sembra oggi essere del tutto svanita. Soprattutto sul versante cinematografico la produzione attuale, se misurata sulla capacità di dare voce ai subalterni e di condizionarne positivamente la vita politica, non può essere in alcun modo confrontata a quella dell'immediato dopoguerra neorealista. Il cinema e la letteratura non sono infatti più luoghi di costruzione delle identità collettive, né lo spazio di elaborazione della coscienza delle classi popolari. Allo stesso modo i soggetti della sinistra eredi del movimento operaio hanno progressivamente abbandonato del tutto l'ambizione di mutare radicalmente la società o comunque di trasformarla. Soprattutto nel Mezzogiorno d'Italia sono sorte parallelamente nuove forme di subalternità e di dominio.

La cultura e le arti non sono più un elemento di mediazione. La lotta politica si svolge secondo forme e pratiche persino più arretrate di quanto accadesse all'inizio del Novecento con il sindacalismo criticato da Gramsci. Di fatto, dopo la scomparsa dei soggetti che si richiamavano al movimento operaio e il ripiegamento antipartitico delle sinistre antagoniste, in Italia e in Europa la socialdemocrazia è progressivamente stata risucchiata nell'orbita dell'ideologia neoliberale, che limita l'azione politica alla mera gestione della cornice esterna del campo sociale in cui si confrontano molteplici e isolate libertà individuali. Le istanze del privato prevalgono dunque sul pubblico, quest'ultimo orientato ormai a diventare un semplice dispensatore di servizi e non più il luogo di costruzione della comunità. Conseguentemente, nella società neoliberale la cultura e le arti perdono ogni facoltà di mediazione dei processi storici.

Non sorprende allora che in questo frangente la letteratura e il cinema, anche fra gli autori più impegnati, abbiano smarrito qualsiasi presa sui processi storico-politici. In Italia in particolare non esiste più una cultura nazionale-popolare e parallelamente è scomparsa dall'ordine del giorno la questione meridionale, nonostante l'aggravarsi del divario economico e sociale tra Nord e Sud. Oggi,

27. AtтAL, Frédéric, Histoire des intellectuels italiens an XXe siècle. Prophètes, philosophes et experts, Paris, Les Belles Lettres, 2013, pp. 285-309 e 113-137.

28. Cfr. Togliatti, Palmiro, La politica culturale, Roma, Editori Riuniti, 1974, p. 198. 
seciè consentito un gioco di parole, domina invecela cultura "nazionalpopolare"29, ovvero la cultura che rinuncia a qualsiasi rapporto pedagogico di elaborazione della coscienza di classe e che degrada i subalterni, offrendo loro una rappresentazione macchiettistica. È esemplare a questo proposito tutto il filone della recente commedia meridionalistica, vera e propria degenerazione della commedia all'italiana del passato. Film come Benvenuti al Sud (2010) con Claudio Bisio, Quo vado con Checco Zalone, Qualunquemente con Antonio Albanese assecondano lo stereotipo del meridionale pigro, bonaccione, mammone, alla ricerca del posto fisso, in un mondo in cui occorre essere invece svelti, dinamici, competitivi e autonomi.

Un caso assai controverso è quello di Roberto Saviano, autore tra i più presenti nella scena mediatica italiana anche per il potente sostegno dell'industria culturale, come la Mondadori, la Rai, il Gruppo Editoriale l'Espresso e più recentemente il Gruppo Feltrinelli ${ }^{30}$. Sul piano dell'impegno la sua produzione è spesso animata dalla sterile retorica della società civile del Sud ${ }^{31}$, che rimarca la divaricazione tra pensiero e prassi dell'attuale età neoliberale. È tuttavia interessante l'operazione di riadattamento cinematografico del suo romanzo d'esordio, Gomorra, condotta da Matteo Garrone, regista tra i più valenti in Italia. Si tratta per la verità di un film (2008) che presenta numerose differenze con il romanzo sia sul piano del contenuto - la scena si svolge con poche eccezioni solo in Campania - sia sul piano formale. In particolare è estremamente significativa la rinuncia alla prima persona che Saviano usa per filtrare il mondo criminale che ruota intorno alla camorra. Nel suo film Garrone cerca al contrario di occultare la macchina da presa e l'istanza enunciante che la governa nel tentativo di limitare il più possibile le mediazioni che possano interferire $\mathrm{e}$ ridurre la trasparenza tra realtà e immagine. In questa forma di realismo si potrebbero individuare degli aspetti comuni col Neorealismo. In realtà, rispetto

29. La differenza tra "nazionale-popolare" e "nazionalpopolare" è al centro di numerosi studi, tra i quali Luporini, Maria Bianca, "Alle origini del «nazionale-popolare»", in Baratta, Giorgio, Catone, Andrea (a cura di), Antonio Gramsci e il "progresso intellettuale di massa", Milano, Unicopli, 1995, pp. 43-51; Durante, Lea, "Nazionalepopolare", in Frosini, Fabio, Liguori, Guido (a cura di), Le parole di Gramsci. Per un lessico dei "Quaderni del carcere", Roma, Carocci, 2004; Schirru, Giancarlo, "Nazionalpopolare", in Giasi, Francesco, Gualtieri, Roberto, Pons, Silvio (a cura di), Pensare la politica. Scritti per Giuseppe Vacca, Roma, Carocci, 2009, pp. 239-253.

30. Dal Lago, Alessandro, Gli eroi di carta. Il caso Gomorra e altre epopee, Roma, Manifestolibri, 2010.

31. Prospero, Michele, Il libro nero della società civile, Roma, Editori internazionali riuniti, 2013. 
ad esso è del tutto assente quel carattere messianico che possiedono le immagini del cinema di Rossellini, De Sica e Visconti. Il mondo popolare e subalterno del Mezzogiorno italiano ripreso da Garrone è senza speranza, abbandonato ai margini della storia. Quello che però è paradossale è che la forza dello stile di Garrone è tutta in negativo: nel cercare il massimo della trasparenza il suo realismo esibisce l'ottusità del reale e insieme ad essa le attuali condizioni di impossibilità di quel nesso tra sentire, comprendere e sapere ricercato negli anni Quaranta e Cinquanta attraverso Gramsci. Tutto questo è ben evidente nella costruzione narrativa dei personaggi. La loro interiorità è sempre inafferrabile, irraggiungibile, schiacciata da un determinismo che non lascia possibilità di riscatto. È esemplare a questo proposito la scena dell'unico tentativo di ribellione presente nel film, quando Roberto - nome forse scelto in omaggio allo scrittore campano - decide di non seguire più Franco e la sua attività illegale di avvelenamento del territorio della provincia di Caserta con i rifiuti importati nel Nord. La sua scelta è individuale, incapace di rovesciare la logica criminale che domina in Italia, ma soprattutto di divenire parte di un processo politico più ampio in grado di fare il "passo in avanti" gramsciano menzionato in precedenza.

L'impossibilità di un'uscita collettiva, e dunque politica, dall'oppressione che domina il Sud si riconosce in maniera altrettanto efficace in un secondo film di Garrone, Reality, in cui il protagonista - in una Napoli in cui elementi di vita quotidiana premoderni e postmoderni si intrecciano tra loro - sogna di essere scelto per entrare nella casa del Grande fratello e vive come se la sua quotidianità fosse continuamente osservata dall'esterno dai selezionatori di Mediaset, come se già vivesse in un reality show. Tra i tanti elementi di interesse del film, quello che colpisce è lo svuotamento culturale operato sulle classi popolari al quale nemmeno Napoli, nonostante la sua grande tradizione ${ }^{32}$, sembra sfuggire. Nei termini di de Martino, l'esaurimento della propria tradizione comporta lo smarrimento di quell'universo di valori e pratiche significanti collettive nel quale trova risoluzione il dramma individuale, ovvero la perdita della presenza ${ }^{33}$. Quello a cui porta la cultura del Grande fratello è infatti un trascendimento senza ethos, una risoluzione del dramma individuale senza valore simbolico

32. Cfr. Luglio, Davide, Il Sud come "forma di vita". L'esempio di Napoli nell'opera di P.P. Pasolini, nel presente volume, pp. 81-90.

33. de Martino, Ernesto, Il mondo magico: prolegomeni a una storia del magismo [1948], Torino, Bollati Boringhieri, 1973. 
collettivo, che incorpora il singolo, le sue aspirazioni e i suoi desideri nella macchina consumistica del capitale di cui diviene prodotto e merce.

Nei due film di Garrone il mondo popolare e subalterno è tornato alla condizione di disgregazione descritta da Gramsci all'inizio Novecento: è classe in sé, senza essere capace di divenire classe per sé, senza cioè essere capace di avviare un lavoro di autocoscienza politica. Rispetto al secolo scorso, la maggiore differenza è che il tema dell'organizzazione partitica è stato abbandonato anche dai presunti soggetti progressivi. La stessa nozione di popolo è stata rigettata da molti intellettuali, perché considerata foriera di populismo. In realtà, come mostrano i fatti più recenti, il populismo è dato dalla rinuncia alla politica organizzata capace di elevare le masse, disgregate e deprivate di un'autonomia culturale, a soggetto della storia antagonista del neoliberismo ${ }^{34}$. Recuperare la lezione di Gramsci sul Sud significa in questo senso recuperare l'idea che il popolo sia una costruzione politica ottenuta anche attraverso lo stretto concorso della cultura e della mediazione che dal sentire porta al comprendere e dal comprendere porta al sapere. Il Mezzogiorno d'Italia, oggi come in passato, proprio perché esibisce in maniera esemplare le contraddizioni del capitalismo attuale, può e deve essere il luogo di partenza di un nuovo riscatto sociale dei subalterni.

Paolo Desogus

Université Paris-Sorbonne (ELCI-EA 1496)

34. Formenti, Carlo, La variante populista. Lotta di classe nel neoliberismo, Roma, DeriveApprodi, 2016. 
